\title{
The Value and the Representation of the Concept - "Dream" in the Lexical-Semantic System and Discourse of English Language
}

\author{
Tatia Chiradze \\ The University of Georgia
}

\begin{abstract}
The article is dedicated to the study of the value meaning of the concept "dream" in English linguistic thought. The study is carried out within the frames of the prominent anthropocentric paradigm of modern linguistics. Dream as a complex and multifaceted reality of the human inner world is one of the existentially significant aspects of the functioning of the human psyche.

The axiological attitude towards the world is of a purely anthropocentric nature. It is in this space that man creates values and it is in this space that values are internalized. Universality is a certain result of the language anthropocentrism and orientation by language towards man and universe. The linguistic sign of the "dream" value is a conventional means of understanding existing knowledge about the inner world of man. The dream, as a powerful and effective tool of self-realization, is the basis of personal development idea in the Western world. In the language system, the linguistic sign "dream" is understood as one of the core value elements of the English linguo-cultural tradition.

Thus, positioning as a value in the English language consciousness, the "dream" is characterized by sharply expressed ambivalence of evaluation which means the following: A dream as an action of an individual or a society, is evaluated both positively and negatively.
\end{abstract}

Keywords: Dream; Lexical-Semantic System, Linguistic Thought, Linguo-Cultural Analysis.

\section{Introduction}

Dream as a complex and multifaceted reality of the human inner world is one of the existentially significant aspects of the functioning of the human psyche. As a determined, intellectual process and a special state of the individual's consciousness, the dream acquires the status of a phenomenon of the inner world that functions according to its own laws. A fragment of linguistic thought having a truly anthropocentric nature defines not only the 


\section{Social science, Humanities \& Education}

various features of the human psychosocial portrait but also particular aspects of human activity and value orientations as well.

Encompassing a whole repertoire of thought, feelings and emotions, the dream appears to be one of the most essential fragments of the semiosphere of the human inner world and at the same time extremely complex and tangible as an abstraction.

\section{Body of paper}

Value is one of the basic categories along with causality, emotional expression, and other egocentric categories. Value is formed against the background of the interaction of those concepts which construct a continuous continuum from a semantic point of view. Valuecontent field represents the orientational and motivational core of the individual determining the specifics of the relations on the basis of interaction between "man and world".

The essence of value - "dream" belongs to the series of universals. Universality is a certain result of the language anthropocentrism and orientation by language towards man and universe. However, it is impossible to absolutize a given quantity, since the perception and conceptualization of the man's inner world is in correlation with the conditions of human existence in particular ethnos (Wierzbicka, 1999, pg. 291-293). The root of linguistic allomorphism is in the cultural tradition, its existence is evident against the background of extralinguistic factors (Wierzbicka, 1997). Thus, culture represents that essential core in connection with national specifics of the worldview reflected in linguistic forms are realized. Therefore, the linguistic sign of the "dream" value is a conventional means of understanding existing knowledge about the inner world of man. The stated knowledge emerges at the intersection of language, thought and culture and ensures their unity in search.

The discussion of the dream phenomenon from a cognitive perspective addresses the issue of modeling the whole human form. The study explicates the ethno-specificity of cognitive interpretation of "dreams" as one of the existentially important fragments of the linguistic icon of the universe. The obtained data adds completeness to the existing notion of typical characteristics of English-speaking linguistic and cultural community.

Values are revealed and realized through evaluation. Man evaluates and divides the world within axiological type of reasoning. Mechanism of adaptation to the human world lies precisely in this action of man. Linguistic evaluation is a special kind of cognitive activity which is the result of realizing experience. It is based on scientific knowledge as well as facts of initial consciousness. Thus, the continuous process of the world comprehension is carried out through evaluation.

The vertical value occupies a central place in the value of the world view reflecting the ethnospecificity of the division of the world by human consciousness. The value essence of dream is placed at the top of the English world hierarchy of value icon. The dream, as a powerful and effective tool of self-realization, is the basis of personal development idea in the Western world. The high axiological status of the value essence of the dream is determined by various parameters among which the ability of the dream to motivate the subject for action and change the world for the better is dominant. 


\section{Social science, Humanities \& Education}

In the language system, the linguistic sign "dream" is understood as one of the core value elements of the English linguo-cultural tradition. The axiological perception of the dream phenomenon is revealed on the level of conceptual ontology: In semantics of linguistic sign "dream", the elements of the comprehension process and the results are revealed. The definitions fixed in the dictionary of the linguistic sign "dream" form an idea about the conceptual core of value. The following conceptual signs form the basis for understanding the phenomenon of dreams by the collective consciousness: conscious mental activity, positive emotional perception, vague state of mind, hope / aspiration, a wild fancy or hope, something ideal.

The linguistic sign "dream" is highly significant which indicates to the multicomponent nature of value content. The content side of the latter includes elements of various fundamental personal structures (desire, hope, purpose, imagination). The analysis of the axiological meaning of the dream, taking into account the adjacent values (reverie; wish / desire; hope; aspiration / ambition / goal; fantasy / fancy), revealed that:

1. The value synthesis is the basis of the study of the phenomenological peculiarities of axiological coordinates.

2. The cognitive specificity of a dream is determined by linguistic (Polysemy and ambivalence, antonyms within the framework of statics / dynamism and reality / unreality) and pragmatic (dominant having vivid value: orientation towards well-being and high value status of the object, high degree of understanding and sustainability) factors.

The content of the prototype situation of a dream in English linguistic thought includes different types of (axiological, intentional, emotional, aesthetic) components: "Dream Subject", "Dream Object", "Subjectivity", "Stereotypicality", "meaningfulness", "firmness", "positive emotional coloring”, “dynamism / staticity", "reality / unreality", "prospectivity", "idealization".

The analysis of empirical material revealed that the dream is often conceived as a means of expanding existential frameworks by stepping out of the realm of real life, ignoring the negative aspects of existence. Thus, dreams are often based on a synthesis of reality elements and their modifications.

One of the fundamental features of thinking is revealed in a dream - mental expansion of the horizon of world cognition which allows one to penetrate into the field beyond of human experience. The value of the phenomenological essence of a dream is synthetic, since it integrates elements of the emotional state of the psyche and the will of the individual. The basis of the value significance of the dream phenomenological essence is created by positive emotions towards the object, which leads to a positive evaluation.

In the English language thought, the dream is anthropomorphic in nature - its conceptualization is carried out in the prism of those properties characterizing a person as an individual with an organized mental apparatus and social relationships.

The causal analysis of the material revealed the close connection of the value of dreams with the problems of human existence. The linguistic structure of the dreams causal space is based on invariant concepts (reality, frustration, wish, emotion). The analysis of discourse fragments 


\section{Social science, Humanities \& Education}

15 - 17 December, 2020

Berlin,Germany

revealed that the reason for the dream lies within the space of the real world and in the frustration with the real world.

Cognitive analysis of the material revealed the fact that the concept of "dream", as a reality of man's inner world, arises as a result of dissatisfaction and frustration with reality.

The semantic structure of the "dream" axiological coordination is a completely open system consisting of a complex of versions of individual-personal conceptualization of the man's multidimensional inner world. A rich semantic polyphony of the axiological significance of the "dream" is revealed in the context of the discursive realization of its linguistic sign. The linguistic sign "dream" has many individual-personal meanings in its speech hypostasis, the content of which is much richer and more diverse than the meanings that make up the conceptual core of the value of the dream.

The discursive actualization of the linguistic sign "dream" reveals the possibility of the existence of new frontiers of the dream phenomenon cognitive conceptualization in English Language. The gnosiological dimension forms one of the most important perspectives for cognitive conceptualization of the dream axiological coordinate. The "dream" is conceived as a permissible limit of the world cognition beyond which an enigmatic space begins.

The interest of the individual to expand his own existential framework, to find a "better world" in which he feels happy is highlighted in terms of the value to be studied. The dream implies a constant search for harmony and perfection in a world full of contradictions.

On the other hand, the true value of a dream lies in its ability to satisfy demands in positive emotions. Playing the role of an important regulator of various mental states, the "dream" equates the negative aspects of the world through virtual realization of needs and goals to the feelings of weakness and powerlessness of human beings.

The evaluative dimensions of the dream axiological significance depend entirely on the reference point where the "I-subject" as a figure with a given emotional experience, confronts the "other" and evaluates it from the position of mind. The "other" is assessed within a strict moral ethical framework of behavior from which deviation leads to individualism, "strangeness".

Historically established cultural dominants of behavior which are based on stereotypical value orientations of a given linguistic collective are found in the English Linguocultural Space. Representing an active position of life, the Anglo-Saxon type of culture considers the idea of advancement, progress and innovation as the basis of personal and social development. This is why the above-mentioned culture considers dreaming and daydreaming as unproductive ways to spend time.

Thus, positioning as a value in the English language consciousness, the "dream" is characterized by sharply expressed ambivalence of evaluation which means the following: A dream as an action of an individual or a society, is evaluated both positively and negatively. 
$4^{\text {th }}$ International Conference on

\section{Social science, Humanities \& Education}

15 - 17 December, 2020

Berlin,Germany

\section{Conclusion}

A complex study of the value of dreams was based on the postulates and principles of linguoculturology, axiology, cognitology, philosophy, psychology. The complexity of the methodology provided the opportunity for a multifaceted analysis of one of the most important fragments of the human inner world and the full revelation of the semantic potential of this fragment.

Thus, the analysis of the material allows the formulation of certain conclusions: The value of the dream is an integral part of the human inner world semiosphere and the axiological dominant of the English linguistic-cultural tradition. The "dream" is one of the foundations of human existence which determines significant aspects of interaction with the human world.

\section{References}

Wierzbicka, A. (1997). Understanding cultures through their key words. Oxford University Press.

Wierzbicka, A. (1999). Emotions across Languages and Cultures. Cambridge University Press 